\title{
Stefan Zweig, l'Européen antipolitique : une lettre inédite à René Schickele
}

Jacques Le Rider

\section{(e) OpenEdition \\ 12 Journals}

Édition électronique

URL : http://journals.openedition.org/rbnu/645

DOI : $10.4000 /$ rbnu. 645

ISSN : 2679-6104

Éditeur

Bibliothèque nationale et universitaire de Strasbourg

Édition imprimée

Date de publication : 1 novembre 2017

Pagination : 12-15

ISBN : 9782859230678

ISSN : 2109-2761

Référence électronique

Jacques Le Rider, « Stefan Zweig, l'Européen antipolitique : une lettre inédite à René Schickele », La Revue de la BNU [En ligne], 16 | 2017, mis en ligne le 01 juillet 2019, consulté le 10 décembre 2020. URL : http://journals.openedition.org/rbnu/645; DOI : https://doi.org/10.4000/rbnu.645

\section{(c) (i) (2)(2)}

La Revue de la BNU est mise à disposition selon les termes de la Licence Creative Commons Attribution - Pas d'Utilisation Commerciale - Partage dans les Mêmes Conditions 4.0 International. 
Wien MS 6936

$22 \cdot 3 \cdot 35$.

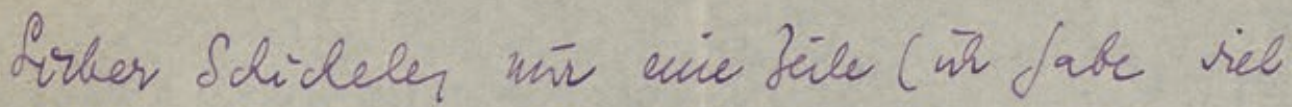
$z_{n}-\operatorname{hin} 1+$ rezt sie nor inceuer fewer Sedaule, ri fitochift lerauifigeben." Es ware fert is ber hwaig eir Consern unpolitiadester art ta, ber ehras atculukes rortat nit Eorkerrint waire, Sie als vollij niaffareigen ats wale aunt qüt torierten feiter uner dotlij statfargijer wir nupolitaden Rera fi gewicuen. oqgea Sie uir uit anem Zion, ob it Sie ben berrefteartero therren berenkigt bin, in hordag ji-Gringea. 2h bir work 10 Taje tiè in Gren, stotel Regina beefrisplag.

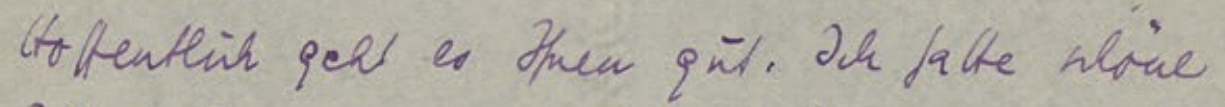
fir an America, meni mic boucect left Levein 4hr it waie froh, hatue ar usal gerare ot

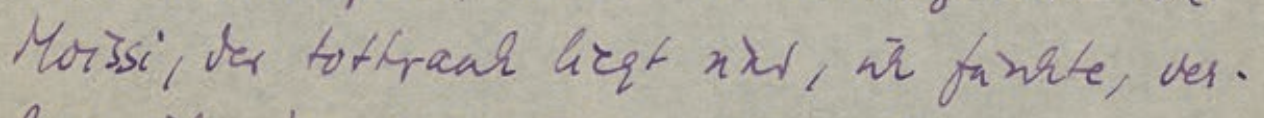
erea it. Herghen sur allew ON

$\therefore$ Sefaumeis 


\section{AUTRICHE \\ STEFAN ZWEIG, L'EUROPÉEN ANTIPOLITIQUE : UNE LETTRE INÉDITE À RENÉ SCHICKELE \\ PAR JACQUES LE RIDER}

Wien, 22. 3.35

Lieber Schickele, nur eine Zeile (ich habe viel zu tun) - reizt Sie noch immer jener Gedanke, die Zeitschrift herauszugeben? Es wäre jetzt in der Schweiz ein Concern unpolitischester Art da, der etwas Ähnliches vorhat und hocherfreut wäre, Sie als völlig unabhängigen und wohl auch gut dotierten Leiter einer völlig unabhängigen und unpolitischen Revue zu gewinnen. Sagen Sie mir mit einem Wort, ob ich Sie dem betreffenden Herren berechtigt bin, in Vorschlag zu bringen. Ich bin noch 10 Tage hier in Wien, Hotel Regina Dollfußplatz.

Hoffentlich geht es Ihnen gut. Ich hatte schöne Zeit in America, mein Buch kommt jetzt heraus und ich wäre froh, käme ich nicht gerade von Moissi, der totkrank liegt und, ich fürchte, verloren ist. Herzlichst Euch allen Ihr

\section{Stefan Zweig}

\section{Vienne, 22 mars 1935}

Cher Schickele, juste une ligne (j'ai beaucoup à faire) - Êtes-vous toujours tenté par l'idée d'éditer la revue ? Il y aurait maintenant en Suisse un groupe industriel de nature tout à fait apolitique qui a un projet semblable et serait très heureux de vous engager comme directeur entièrement indépendant, et sûrement aussi bien payé, d'une revue entièrement indépendante et apolitique. Dites-moi d'un mot si vous m'autorisez à vous proposer au monsieur concerné. Je suis encore pour dix jours ici, à Vienne, hôtel Regina, place Dollfuss.

J'espère que vous allez bien. J'ai fait un beau séjour en Amérique, mon livre sort maintenant et je serais heureux, si je ne sortais pas de chez Moissi qui garde le lit, mortellement malade, et qui est perdu, je le crains. Très cordialement à vous tous, votre

\section{Stefan Zweig}


En mars 1935, après un séjour à New York en janvier, suivi d'une décade à Londres et d'un passage à Salzbourg, Stefan Zweig se rend à Vienne, où il s'installe à l'hôtel Regina, pour se concentrer sur la correction des épreuves de sa nouvelle biographie historique, Marie Stuart, publiée chez l'éditeur viennois Herbert Reichner, et pour revoir sa mère qui se remet d'une grave maladie. La fin de cette lettre du 22 mars fait allusion à l'acteur Alexandre Moissi, mort le 23 mars 1935 à la veille de son cinquante-sixième anniversaire. Luigi Pirandello avait demandé à Zweig de traduire sa pièce Non si sa come (On se sait comment, Man weiß nicht wie), où Moissi devait jouer le rôle principal, et les répétitions devaient commencer à la fin mars au Volkstheater de Vienne. Mais Moissi a contracté une pneumonie à Rome, au cours d'une tournée en Italie, et le sombre pronostic de Zweig est confirmé dès le lendemain.

C'est à la fin de 1917, à Zurich, que Stefan Zweig a fait la connaissance de René Schickele qui dirigeait depuis 1915 la revue littéraire, qu'il avait ouverte aux idées pacifistes, Die weißen Blätter (Les Pages blanches), éditée en 1916 et 1917 par Max Rascher, chez qui Schickele fut responsable également de la collection Europäische Bibliothek. Zweig a revu Schickele en décembre 1934: au cours de son séjour à Nice, il est allé à Sanary-sur-mer, où se retrouvent à l'époque beaucoup d'exilés allemands. Schickele, installé à Sanary depuis mars 1934, lui a certainement parlé de son souhait de fonder une revue européenne à laquelle il pensait donner le titre de Verteidigung des Westens ${ }^{1}$ - ce qui aurait présenté l'inconvénient de prêter à confusion avec le titre choisi par Henri Massis pour son essai de 1927 La Défense de l'Occident, repris dans un article publié dans Le Temps le 4 octobre 1935, «Pour la défense de l'Occident ".

Depuis les années 1920, Zweig plaide pour l'unification européenne, seule chance de conjurer la menace d'une nouvelle guerre et d'arrêter l'affrontement des impérialismes. Son idéalisme, son refus de la Realpolitik, son aspiration à penser l'Europe sous le signe d'une éthique cosmopolite et à renouer avec l'universalisme humaniste s'opposent à ce qu'on appelle aujourd'hui la mondialisation capitaliste. Dans sa vision utopique, l'unité européenne devrait être d'abord spirituelle. Lors de son premier séjour au Brésil, le 27 août
1936, à Rio de Janeiro, il donnera une dimension encore plus vaste au rapprochement culturel des peuples et des nations et parlera (en français) de l'unité spirituelle du monde.

Zweig, comme Schickele, considère la publication de revues comme la concrétisation de la " république des lettres " européenne et universelle, à l'édification de laquelle il veut contribuer. Dans son texte de 1932 La Désintoxication morale de l'Europe, il insiste sur la nécessité « de réaliser l'union culturelle de l'Europe avant son union politique, militaire et financière qui se heurte aujourd'hui encore à des tendances farouchement opposées. Un organe de presse commun aux Européens, une revue ou mieux encore un journal quotidien, contribuerait éminemment à une telle entente : publié dans toutes les langues d'Europe avec le même contenu, il aurait pour raison d'être de s'interdire tout propos susceptible d'accroître les malentendus et de signaler toutes les possibilités de renforcer les liens et la compréhension mutuelle. Ce serait en somme une publication, journal ou revue, positive, optimiste, roborative, qui montrerait à la jeune génération, dans tous les pays, la mission [...] qui se propose à elle ${ }^{2}$.

Au moment où il rencontre Schickele à Sanary, en décembre 1934, les projets de revue se bousculent dans l'imagination de Zweig. Le 30 décembre, de l'hôtel Westminster à Nice, il écrit à son éditeur new-yorkais Ben Huebsch, le directeur de Viking Press, pour lui rappeler son souhait de créer une revue juive ${ }^{3}$. Mais il s'agit certainement d'un autre projet que celui dont il est question avec Schickele. Dans cette lettre à Ben Huebsch, Stefan Zweig précise qu'il souhaite parler du projet avec le romancier américain Ludwig Levisohn.

On est frappé par l'insistance avec laquelle Zweig, dans sa lettre à Schickele, souligne le caractère indépendant et apolitique de la revue dont la direction pourrait lui être confiée. Depuis le pénible épisode de sa rupture avec Klaus Mann, les réticences de Zweig à s'engager aux côtés des intellectuels antifascistes se sont transformées en véritable phobie de la politique. Une telle attitude ne pouvait que déconcerter et parfois même indigner ses contemporains. Après avoir accepté, en mai 1933, de collaborer à la revue antinazie animée par Klaus Mann Die Sammlung, et annoncé l'envoi d'un chapitre de son Érasme, Zweig avait changé d'avis et, le 11 septembre 1933, il avait demandé à Klaus Mann de 
retirer son nom de la liste des collaborateurs de sa revue, puis écrit à son éditeur Anton Kippenberg, directeur des éditions Insel, pour l'assurer de son hostilité à la ligne politique de la revue Die Sammlung. Cette lettre de Zweig, publiée sans son accord le 14 octobre 1933 dans le journal de la librairie allemande contrôlé par les nazis, était apparue comme un geste d'autant plus affligeant qu'il était vain de sa part d'espérer un traitement de faveur qui lui permettrait de sauvegarder ses intérêts sur le marché du livre allemand.

Depuis lors, Zweig n'est plus seulement apolitique, mais bien antipolitique au sens que Nietzsche donne à ce mot dans Ecce homo : «Il ne m'en coûte point d'être un 'bon Européen', [...] moi qui suis le dernier Allemand antipolitique ${ }^{4}$. Quelques jours avant sa lettre à Schickele, il écrivait le 18 mars 1935 à Romain Rolland, l'ami pacifiste devenu partisan de l'URSS : «La politique nous abrutit. Elle est tellement dégoûtante, absurde, qu'on ne se sauve qu'en crachant sur elle $»^{5}$. Comme on l'a dit, cette attitude déconcertait et, souvent même, indignait ses contemporains. Mais on comprend mieux aujourd'hui, au moment où les projets d'approfondissement de l'Union européenne semblent bloqués, que l'idéalisme européen de Stefan Zweig, pour qui l'unité culturelle et spirituelle des Européens était la condition préalable à leur union économique et politique, s'enracinait dans son expérience vécue depuis la crise de 1929 : en temps de crise, les tentations protectionnistes et nationalistes reviennent au galop et l'idée européenne elle-même est en péril. C'est alors qu'on se dit qu'il aurait fallu commencer par la consolidation de son « unité spirituelle ».

\section{NOTES}

1- La Défense de l'Occident. Voir Maryse Staiber, L'« exil » de René Schickele (1932-1940), thèse de doctorat, Strasbourg, 1989, p. 32

2- Zweig, Stefan, «La désintoxication morale de l'Europe », in idem, Appels aux Européens, trad. et présentation de Jacques Le Rider, Paris, Bartillat, 2014, p. 71-107, citation p. 97 sq.

3- Zweig, Stefan, Correspondance (1932-1942), trad. Laure Bernardi, Paris, Grasset, 2008, Librairie générale française - Le Livre de poche, 2010, p. 156 sq. Sur le projet de création d'une revue juive, qui ne sera pas réalisé, cf. Jeffrey B. Berlin, « Unveröffentlichte Briefe über Stefan Zweigs USA-Reise im Jahre 1935 und sein Projekt einer jüdischen Zeitschrift ", in Stefan Zweig lebt. Akten des 2. Internationalen Stefan Zweig-Kongresses in Salzburg 1998, Stuttgart, Heinz Akademischer Verlag, 1999, p. 59-82. Je remercie Klemens Renoldner, directeur du Centre Stefan Zweig de Salzbourg, pour les renseignements qu'il m'a communiqués à ce sujet.

4- Nietzsche, Friedrich, Ecce homo, «Pourquoi je suis si sage », par. 3, in idem, Euvres, éd. par Jean Lacoste et Jacques Le Rider, Paris, Laffont (Bouquins), 1993, vol. 2, p. 1120

5- Zweig, Stefan, lettre en français, de l'hôtel Regina, le 18 mars 1935, in idem, Correspondance (1932-1942), op. cit., p. 163-166, citation p. 165 